\title{
Pegaptanib sodium as maintenance therapy in neovascular age-related macular degeneration: the LEVEL study
}

\author{
Thomas R Friberg, ${ }^{1}$ Michael Tolentino, ${ }^{2}$ for the LEVEL Study Group
}

${ }^{1}$ Ophthalmology, University of Pittsburgh Medical Center Eye Center, University of Pittsburgh, Pittsburgh, Pennsylvania, USA ${ }^{2}$ Center for Retina and Macular Disease, Winter Haven, Florida, USA

\section{Correspondence to}

Dr Thomas R Friberg, Retina Service, Eye and Ear Institute, Room 825, 203 Lothrop Street, Pittsburgh, PA 15213, USA: friberg@pitt.edu

Accepted 2 February 2010 Published Online First 14 May 2010

\section{(2) UNLOCK:}

This paper is freely available online under the BMJ Journals unlocked scheme, see http:// bjo.bmi.com/site/about/ unlocked.xhtml

\section{ABSTRACT}

Aim To assess the efficacy of pegaptanib as maintenance therapy in neovascular age-related macular degeneration (NV-AMD) patients after induction therapy.

Methods A phase IV, prospective, open-label, uncontrolled exploratory study including subjects with subfoveal NV-AMD who had had one to three induction treatments 30-120 days before entry and showed investigator-determined clinical/anatomical NV-AMD improvement. Lesions in the study eye were: any subtype, 12 or fewer disc areas; postinduction centre point thickness (CPT) $275 \mu \mathrm{m}$ or less or thinning of $100 \mu \mathrm{m}$ or more (optical coherence tomography); visual acuity (VA) 20/20-20/400. Intravitreal pegaptanib $0.3 \mathrm{mg}$ was administered as maintenance every 6 weeks for 48 weeks with follow-up to week 54 . Booster treatment additional unscheduled treatment for wet agerelated macular degeneration, was allowed in the study eye at the investigators' discretion for clinical deterioration.

Results Of 568 enrolled subjects, $86 \%$ completed 1 year of pegaptanib. Mean VA improvement during induction (49.6 to 65.5 letters) was well preserved (54-week mean 61.8 letters). Mean CPT was relatively stable during maintenance (20 $\mu \mathrm{m}$ increase during the study). Fifty per cent did not receive unscheduled booster treatment to week 54; $46 \%$ did have one such booster (mean 147 days after maintenance initiation)

Conclusions An induction-maintenance strategy, using non-selective then selective vascular endothelial growth factor (VEGF) inhibitors, could be considered for NVAMD. This approach may have particular relevance for patients with systemic comorbidities who require longterm anti-VEGF therapy for NV-AMD.

Treatment of neovascular age-related macular degeneration (NV-AMD) has changed dramatically in the past 5 years with the clinical use of intravitreal agents specifically targeting vascular endothelial growth factor (VEGF). This development, the culmination of more than a decade of work into the pathophysiology of NV-AMD, led to the approval of two drugs as intravitreal therapies: pegaptanib sodium, an RNA aptamer targeting $\mathrm{VEGF}_{165},{ }^{1}$ and ranibizumab, a monoclonal antibody fragment that binds all VEGF isoforms. ${ }^{2}{ }^{3}$ In addition, bevacizumab, a monoclonal antibody related to ranibizumab that also binds all VEGF isoforms, has been used off-label extensively to treat a variety of ocular neovascular conditions. ${ }^{4}$

The optimal utilisation of these agents remains a matter of debate. In pivotal trials, ranibizumab provided a significant improvement in mean visual acuity (VA) compared with control groups in NVAMD patients. ${ }^{2}{ }^{3}$ It has been suggested that these results may be attributed to its mechanism of action in binding all VEGF isoforms. ${ }^{5}$ However, the systemic safety of pan-VEGF agents still concerns some practitioners, and the use of the non-selective VEGF antagonist, bevacizumab, in cancer chemotherapeutic regimens has been associated with an increased incidence of hypertension, bleeding and thromboembolic events. ${ }^{6}$ Whereas the doses employed with intravitreal administration are much lower, systemic exposure is likely given that the aberrant vasculature tends to be particularly leaky. This safety concern is relevant to the NV-AMD patient who is already at increased risk of hypertension, stroke and cardiovascular disease ${ }^{7}$ and, thus, is at greater risk of treatment-related systemic complications, especially because anti-VEGF therapies are generally used on a long-term basis.

Pegaptanib has been used in clinical studies for more than 4 years without the appearance of systemic or ocular safety signals ${ }^{8}$ and has also been examined at doses 10 times greater than those employed clinically without any evidence of an increased risk of systemic adverse effects. ${ }^{10}$ Its positive safety profile has led to attempts to combine both the efficacy of non-specific inhibition with the apparent safety of pegaptanib. In small-scale studies, an initial inductive dose of a non-specific VEGF inhibitor was followed by maintenance therapy with pegaptanib and booster doses of pegaptanib as required. ${ }^{11}{ }^{12}$ Because these studies reported encouraging results, the present exploratory study (Evaluation of Efficacy and Safety in Maintaining Visual Acuity with Sequential Treatment of Neovascular AMD; LEVEL) was undertaken as a larger scale application of this fundamental methodology. In this study, the safety and efficacy of pegaptanib maintenance in patients with NV-AMD who experienced a clinical improvement in disease following an induction phase were assessed.

\section{MATERIALS AND METHODS}

The protocol for this phase IV, prospective, openlabel, uncontrolled exploratory study was reviewed and approved by an institutional review board at each study site in accordance with the guidelines for the conduct of clinical research in the 1964 Declaration of Helsinki. The study is listed on http://www.clinicaltrials.gov (NCT00354445). All study participants provided signed informed consent. 
Eligible subjects of either gender aged 50 years or older diagnosed with subfoveal NV-AMD documented by fluorescein angiography must have had best-corrected VA of 20/20 to 20/ 400 (Snellen) and had one or more but three or less previous treatments (induction phase) for NV-AMD 30-120 days before study entry. Subjects had to have shown significant improvement of exudative maculopathy after the previous induction phase based on clinical and/or anatomical findings, as determined by the investigator. Although there was no specific definition of clinical improvement, there were two criteria for anatomical improvement based on optical coherence tomography (OCT) assessment: either a centre point thickness (CPT) of $275 \mu \mathrm{m}$ or less at study entry or demonstrated retinal thinning of $100 \mu \mathrm{m}$ or greater occurring between the time of the first induction treatment to study entry. NV-AMD lesions of any angiographic subtype and 12 or fewer disc areas (including blood, neovascularisation and scar/atrophy) were entered.

Excluded were the eyes of subjects with subfoveal scarring, subfoveal atrophy or subfoveal haemorrhage in the study eye that was $50 \%$ or more of the total lesion area or one or more disc areas; eyes with significant media opacities, including cataract or a history of any intraocular surgery to either eye within 3 months of trial entry; and a history of posterior vitrectomy, glaucoma surgery or previous therapeutic radiation in the region of the study eye. Eyes with pigment epithelial tears or rips or that had any ocular or periocular infection in the previous 4 weeks were not enrolled. Subjects with other causes of choroidal neovascularisation were also excluded. Additional exclusion criteria included: diabetic retinopathy; history/evidence of severe cardiac disease, unstable angina, acute coronary syndrome, myocardial infarction or revascularisation within 6 months or ventricular tachyarrhythmias requiring ongoing treatment; clinically significant peripheral vascular disease or impaired renal or hepatic function; stroke within the previous 12 months; major surgical procedure within the previous 1 month; any treatment with an investigational agent in the past 30 days; or known serious allergies to the fluorescein dye used in angiography or to the components of pegaptanib sodium. Women of childbearing potential were required to be using two forms of effective contraception during the trial and for at least 60 days following the last dose of test medication.

It is also important to note that we did not, in the study, include patients who were induced with therapy for wet agerelated macular degeneration (AMD) but then were not shifted to pegaptanib maintenance therapy. Nor did we include patients who were not successfully induced with one to three treatments for wet $\mathrm{AMD}$ as per our inclusion criteria.
Figure 1 Mean visual acuity during induction and maintenance $(A)$ and mean centre point thickness during maintenance (B), $\mathrm{N}=568$.

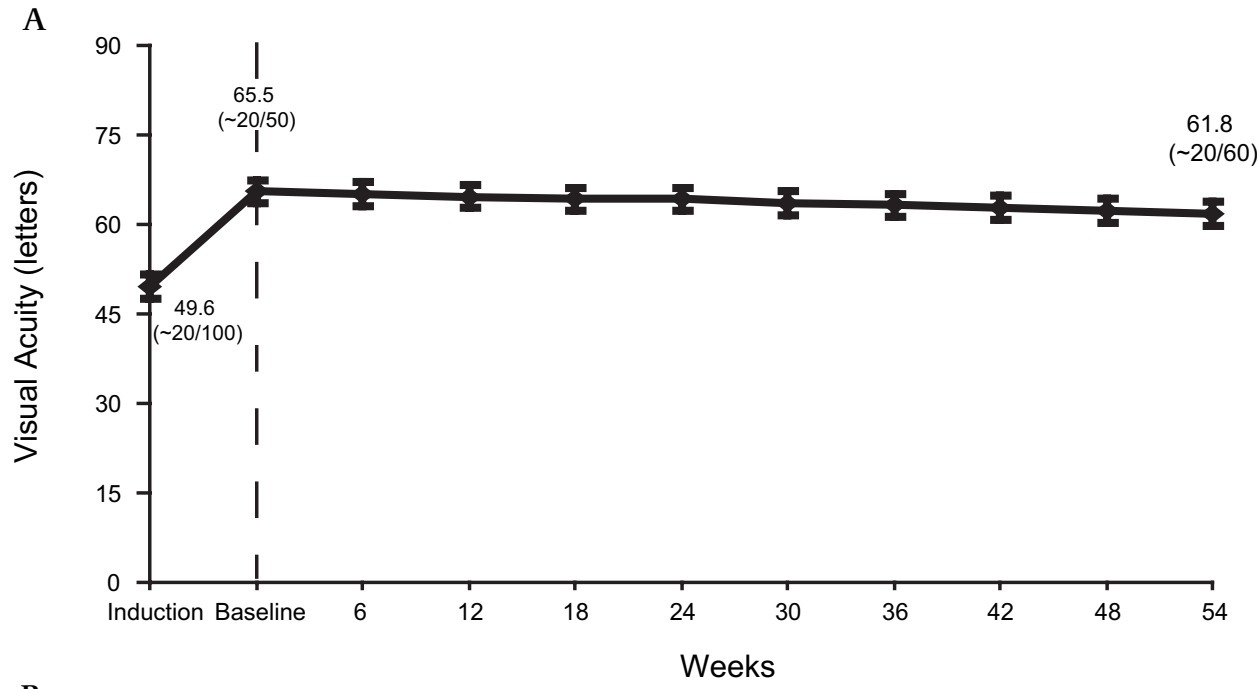

B

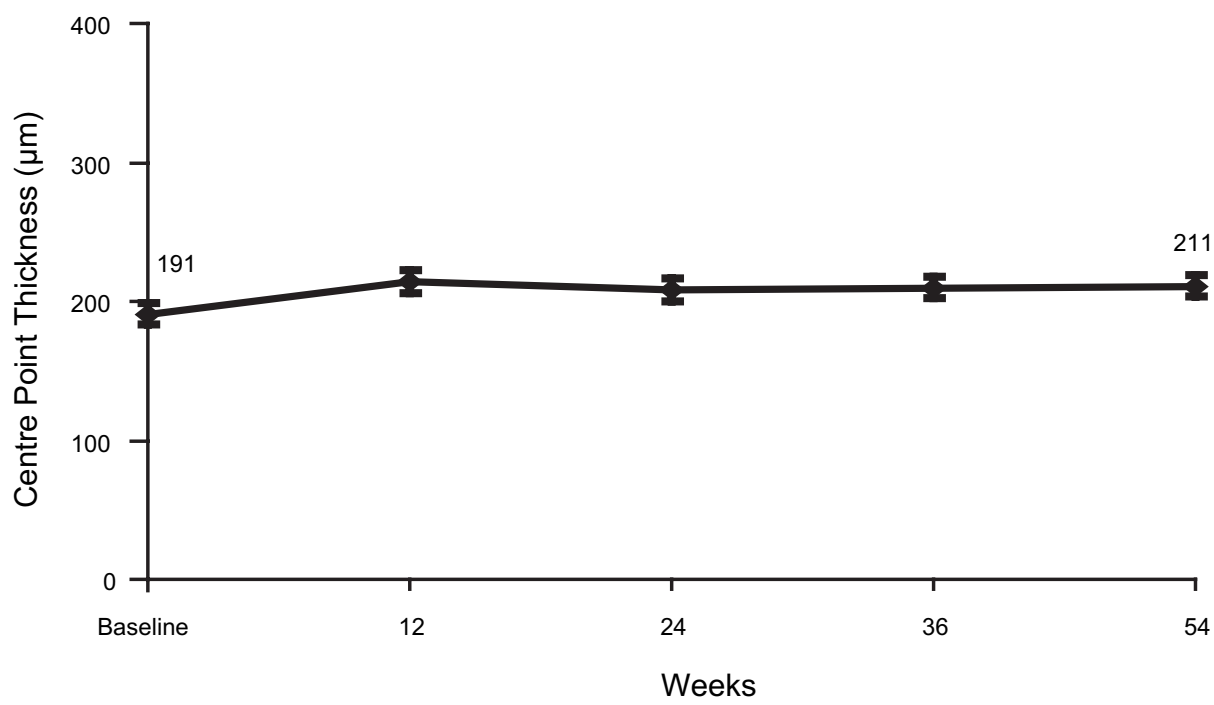


Figure 2 No predictors for booster treatments at baseline, $\mathrm{N}=568$. $0 \mathrm{CT}$, optical coherence tomography.
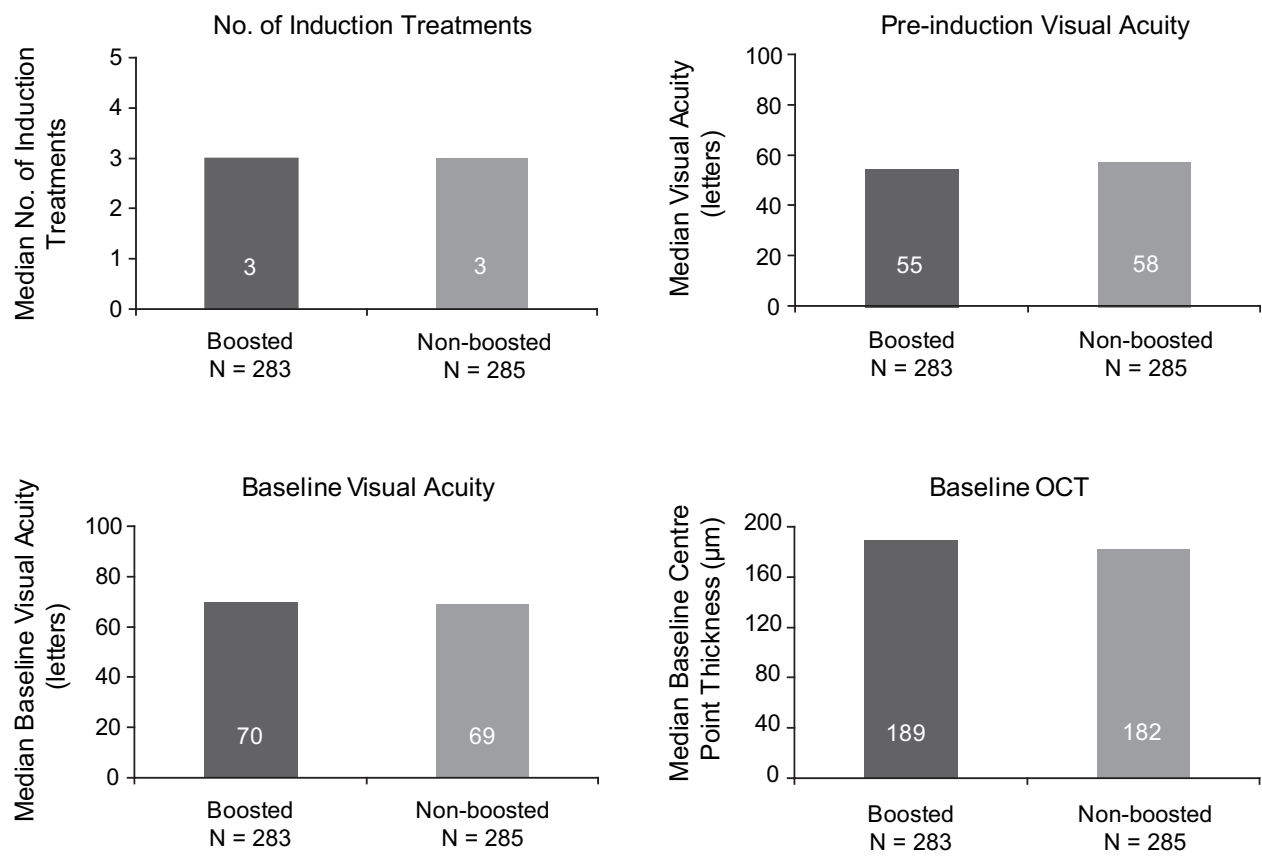

\section{Study assessments and endpoints}

At the baseline visit, which occurred within 7 days before the first pegaptanib injection, a medical and ophthalmological history (including preinduction VA measurements) and baseline assessments were conducted. At day 0 (first pegaptanib injection; may have been baseline day) and weeks 6, 12, 18, 24, 30, 36, 42, 48 and 54, refraction and VA measurements (early treatment diabetic retinopathy scale or Snellen chart) were repeated preinjection, and tonometry (applanation or Tonopen) and an ophthalmological examinations were performed both pre and postinjection. At these visits, tonometry was obtained at least 30 min postinjection. All assessments included both eyes. The study eye was evaluated using OCT preinjection at weeks 12 , 24, 36 and 54, whereas colour fundus photography and fluorescein angiography were performed in both eyes at week 54 . At baseline and all follow-up visits, blood pressure was measured, concomitant medications were recorded and adverse events were documented.

Intravitreal pegaptanib $0.3 \mathrm{mg}$ was administered every 6 weeks for 48 weeks with follow-up to week 54 . Unscheduled treatments with other agents used to treat wet AMD were allowed (booster therapy) at the discretion of the investigator to counter the deterioration of NV-AMD. Regardless of booster treatments, subjects continued to receive intravitreal pegaptanib every 6 weeks unless another anti-VEGF agent was used as booster treatment in which case the pegaptanib injection was not required.

No statistical assessments other than descriptive analyses were prespecified and no formal power and sample size calculations were applied. The proportion of subjects maintaining or gaining vision from baseline to week 54 was nominally considered the primary efficacy endpoint. Secondary endpoints included the proportions of subjects gaining one or more, two and three lines or losing less than three lines of vision at week 54 compared with preinduction, mean VA change from baseline to week 54 and anatomical outcomes seen on fluorescein angiography and OCT. Potential predictors of the need for booster treatment were also evaluated.

Safety endpoints included all adverse events whether spontaneously reported, elicited or observed by investigators. Serious adverse events were defined as those resulting in death or that were life-threatening, resulted in hospitalisation, in persistent or significant disability or incapacity or in a congenital anomaly or birth defect.

The last observation carried forward (LOCF) method was used to impute missing data. Safety assessments included all subjects receiving one or more dose of pegaptanib.

\section{RESULTS}

The LEVEL study began enrolling subjects in the USA in June 2006. A total of 568 subjects from 87 centres was enrolled, and $487(86 \%)$ completed 1 year of pegaptanib maintenance. Subjects were elderly (mean age $78.4 \pm 7.6$ years), the majority were women (338; 60\%), 57 (10\%) were active smokers and 550 $(97 \%)$ were white. Most subjects had at least one preexisting condition, the most common of which were vascular disorders, reported by $433(76 \%)$ subjects, and musculoskeletal/connective tissue disorders, reported by $347(61 \%)$ subjects. With regard to induction, 240 (42\%) received induction with ranibizumab, 207 $(36 \%)$ with bevacizumab, 12 (2\%) with other single agents and 108 (19\%) with multiple agents, including different

Table 1 Ocular adverse events*

\begin{tabular}{lll}
\hline Serious ocular adverse event & No of subjects & $\%$ Per injection \\
\hline Endophthalmitis & $2 \dagger$ & $0.05 \%$ \\
Vitreous haemorrhage & 2 & $0.05 \%$ \\
Conjunctival irritation & 1 & $0.02 \%$ \\
Retinal haemorrhage & 1 & $0.02 \%$ \\
Retinal tear & 1 & $0.02 \%$ \\
Vitreous opacities & 1 & $0.02 \%$ \\
Ocular adverse events reported in $\geq 5 \%$ of study eyes, N (\%) & \\
Punctate keratitis & $73(12.9)$ & \\
Eye pain & $61(10.7)$ & \\
Vitreous floaters & $57(10.0)$ & \\
Conjunctival haemorrhage & $36(6.3)$ & \\
Eye irritation & $34(6.0)$ & \\
Visual acuity reduced & $31(5.5)$ & \\
Macular degeneration & $28(4.9)$ &
\end{tabular}

${ }^{*} \mathrm{~N}=568$ subjects with 4254 total injections.

†One case was a 'possible' endophthalmitis. 
combinations of pegaptanib, ranibizumab, bevacizumab, photodynamic therapy, corticosteroids and transpupillary thermotherapy (data are not available for one subject). Subjects received a mean of 2.6 induction treatments over a median of 15 weeks. Lesions were relatively dry postinduction: the mean CPT was $191 \pm 53 \mu \mathrm{m}, 361$ (64\%) had a CPT of $200 \mu \mathrm{m}$ or less and only 119 (21\%) had a thickness greater than $225 \mu \mathrm{m}$. A mean of eight injections was administered during the study, and $360 / 568$ (63\%) subjects received the maximum of nine pegaptanib injections.

Mean VA improved during induction from 49.6 \pm 21.9 letters to $65.5 \pm 15.3$ letters and was relatively well preserved during maintenance (61.8 \pm 18.9 letters at week 54; figure 1A; LOCF). Preinduction to week 54, 444 (79\%), 372 (66\%), 290 (52\%) and $233(41 \%)$ subjects gained none or more, five or more, 10 or more and 15 or more letters of VA, respectively; 520 (92\%) lost less than 15 letters. (Note that preinduction VA data were unavailable for five subjects who were excluded from these analyses.) Mean VA using observed data (not LOCF data) also improved from baseline to induction $(49.6 \pm 21.9$ letters to $65.6 \pm 15.1$ letters, respectively) and was $61.7 \pm 18.9$ letters at week 54 . Median VA showed a similar improvement during induction
(55-69 letters) and was 66 letters at week 54 . Fifteen per cent of subjects (88/568) had VA assessed with Snellen charts. Excluding those subjects provided similar results on mean VA change over time compared with the overall study population. VA response during pegaptanib maintenance was similar in subjects stratified by induction anti-VEGF agent (bevacizumab vs ranibizumab); best $50 \%$ versus worst $50 \%$ preinduction VA; postinduction VA; postinduction CPT; and one or more letter versus 10 or more letter improvement postinduction. Mean CPT was stable during pegaptanib maintenance (figure $1 \mathrm{~B}$ ).

Approximately half the subjects $(285 / 568)$ did not receive booster treatment to week 54 . Of the 283 subjects receiving booster treatment, 46\% (130/283) required only one such treatment. Among boosted subjects, the mean time between the onset of maintenance therapy and first booster was $147 \pm 92$ days. Neither number of induction treatments, preinduction or baseline VA, nor baseline CPT predicted the need for booster treatment (figure 2).

Pegaptanib was safe and well tolerated. A total of 468 (82\%) subjects reported one or more adverse event, 350 (62\%) experienced an ocular adverse event and $112(20 \%)$ had one or more serious adverse event. During 1 year of follow-up, there was no
Figure 3 Mean intraocular pressure (A) and mean systolic and diastolic blood pressures (B).
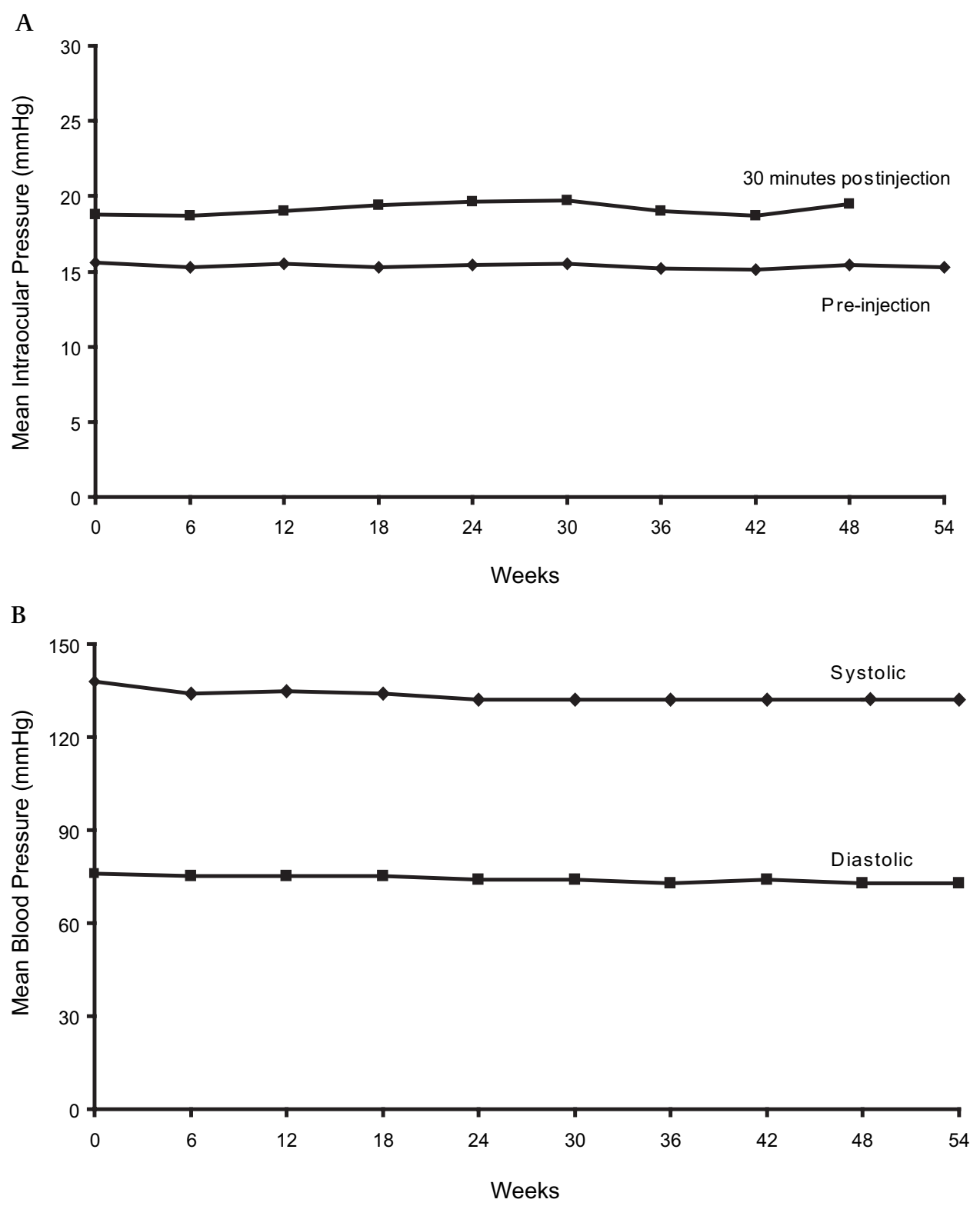
evidence of an increased risk of endophthalmitis, retinal detachment or traumatic cataract when compared with historical data (table 1). ${ }^{8}$ In all, 54\% (304/568) of subjects reported an ocular adverse event; those reported in $5 \%$ or more of study eyes as well as serious ocular adverse events are summarised in table 1. Intraocular pressure was stable across visits both pre and postinjection (figure $3 \mathrm{~A}$ ).

Reports of serious vascular events were rare (table 2). Using the Anti-Platelet Trialists' Collaboration classification, there were six (1.1\%) cardiovascular deaths, five (0.9\%) non-fatal myocardial infarctions and three $(0.5 \%)$ non-fatal cerebrovascular accidents. These rates are similar to those observed in the VEGF Inhibition Study in Ocular Neovascularization (VISION) trials' pegaptanib $0.3 \mathrm{mg}$ group at 1 year (Pfizer Inc, data on file). Minor infections and infestations were the most frequently reported non-ocular adverse events (table 2). Mean systolic and diastolic blood pressures were stable across visits (figure $3 \mathrm{~B}$ ).

\section{DISCUSSION}

The underlying objective of this study was to explore outcomes obtained by combining the safety record of pegaptanib, now validated over 4 years in the continuation of the VISION trials, ${ }^{9}$ after visual gains were obtained during an induction phase. This approach, previously studied in relatively small numbers of patients, ${ }^{11} 12$ has now been supported on a larger scale, with overall visual outcomes including a mean VA improvement of 12.2 letters at 1 year and $41 \%$ of patients gaining three or more early treatment diabetic retinopathy scale lines. Herein, VA gains were provided by the induction therapy, with pegaptanib playing a long-term maintenance role; only half of the patients required a booster treatment, which was given an average of 5 months postbaseline. Anatomical responses, as assessed by OCT, were similarly maintained. As in the VISION trials, ${ }^{8} 9$ pegaptanib was well tolerated, with few ocular or systemic events.

Both ocular ${ }^{13}$ and systemic ${ }^{7}$ adverse effects are potential safety concerns with VEGF inhibition, although systemic

Table 2 Non-ocular adverse events*

\begin{tabular}{lr}
\hline Serious non-ocular adverse event & N (\%) \\
\hline All nervous system & $13(2.3)$ \\
Cerebrovascular accident & $3(0.5)$ \\
Cerebral infarction & $1(0.2)$ \\
All cardiac & $30(5.3)$ \\
Myocardial infarction & $4(0.7)$ \\
Acute coronary syndrome & $1(0.2)$ \\
Coronary artery occlusion & $1(0.2)$ \\
Myocardial ischaemia & $1(0.2)$ \\
All non-ocular haemorrhagic & $2(0.4)$ \\
Haematuria & $1(0.2)$ \\
Other non-ocular adverse events reported in over 2\% of subjects & \\
Hypertension & $43(7.6)$ \\
Urinary tract infection & $26(4.6)$ \\
Sinusitis & $25(4.4)$ \\
Gastro-oesophageal reflux & $21(3.7)$ \\
Pneumonia & $20(3.5)$ \\
Benign prostatic hyperplasia & $10(4.3) \dagger$ \\
Cardiac failure, congestive & $18(3.2)$ \\
Bronchitis & $19(3.3)$ \\
Nasopharyngitis & $16(2.8)$ \\
Headache & $16(2.8)$ \\
Nausea & $15(2.6)$ \\
\hline
\end{tabular}

${ }^{*} \mathrm{~N}=568$ subjects with 4254 total injections.

†Considering only male study population. exposure is likely to be diminished by the higher volume of distribution. The issue of VEGF-dependent ocular homeostasis has not been examined clinically, whereas preclinical data suggest that VEGF plays an essential retinal neuroprotective role in conditions of ischaemia. ${ }^{13}$ In that investigation, the authors found that only $\mathrm{VEGF}_{120}$, the isoform spared by pegaptanib, was required for neuroprotection and that sustained inhibition of all VEGF isoforms led to the progressive loss of retinal ganglion cells. Another ocular structure for which VEGF is believed important is the choriocapillaris. ${ }^{14}$ Preclinical data suggest that intravitreal bevacizumab can lead to the loss of choriocapillaris fenestrations as well as occlusion of the choriocapillaris lumen by thrombocytes and leucocytes. ${ }^{15}$ In addition, recent clinical data have indicated that the intravitreal injection of ranibizumab can induce vasoconstriction of retinal arterioles in $\mathrm{AMD}$ patients. ${ }^{16}$ The authors suggest that this is a downstream effect of lowered VEGF levels because VEGF has been found to induce the synthesis of nitric oxide by endothelial cells ${ }^{17}$ while reduced nitric oxide synthesis results in vasoconstriction (reviewed by Moncada and Higgs). ${ }^{18}$

The potential for systemic complications has received more attention. As discussed in a recent review, ${ }^{7}$ the issue of cardiovascular risk is especially relevant because the AMD population is already at higher risk. Moreover, the role of VEGF is especially complex in the context of atherosclerosis. ${ }^{19}$ As VEGF acts to upregulate the synthesis of tissue factor, the initiator of the coagulation cascade, inhibition of VEGF could theoretically have antithrombotic consequences. However, several other mechanisms are likely to promote thromboses in this setting. ${ }^{19}$ VEGF is an endothelial cell survival factor so that inhibition could favour apoptosis of endothelial cells, leading to their becoming procoagulant. $^{20}$ In addition, VEGF upregulates nitric oxide synthase and ultimately the production of nitric oxide, ${ }^{17}$ a potent anticoagulant. Finally, VEGF has also been reported to destabilise cholesterol plaques, possibly through the inhibition of immature blood vessels forming in them..$^{21}$ Data suggesting higher incidences of non-ocular haemorrhage $e^{22}$ and cerebrovascular accidents ${ }^{23}$ in patients receiving a non-selective anti-VEGF treatment compared with sham injections have been reported. These findings indicate that, for some fraction of NV-AMD patients, minimising exposure to non-selective inhibition is desirable, especially in the setting of this chronic disease that requires treatment for many years. Attempts to maintain the efficacy results obtained with monthly injections of ranibizumab with less frequent dosing have been made with inconsistent results, ${ }^{24} 25$ suggesting that the regular/frequent administration of VEGF inhibitors is necessary to optimise visual outcomes.

In conclusion, the data from this large exploratory study suggest that maintenance therapy with pegaptanib may provide an alternative to prolonged non-selective VEGF inhibition by holding visual gains after induction. As current evidence supports the long-term use of anti-VEGF agents, inductionmaintenance using non-selective followed by selective VEGF inhibitors should be considered for the treatment of NV-AMD. Such an approach has special relevance for patients with cardiovascular comorbidities who require anti-VEGF drugs to manage their NV-AMD. Further study is warranted to confirm these data in broader settings and to refine protocols with a view to minimising the number of treatments required. In addition, we also caution the reader to avoid direct comparisons of the LEVEL trial results with those of other published trials. For instance, in several anti-VEGF studies, the study design included continued monthly injections even if the first 3 months of 
therapy appeared unsuccessful. We do not know how such initial non-responders would fare with LEVEL trial-type treatment, as they were excluded from entry. We do conclude that LEVEL trial-type therapy appears to be a viable option for patients who respond initially to induction therapy and provides an additional practical treatment option for such individuals with wet AMD.

Acknowledgements Editorial assistance was provided by Jane Murphy, PhD, of Zola Associates, Englewood Cliffs, NJ; trial management was provided by Patrick Healy, MPH, of Trial Runners, Dickinson, ND; and statistical analysis was provided by the International Drug Development Institute, Louvain-a-Neuve, Belgium.

Funding This study was sponsored by Eyetech, Inc and OSI Pharmaceuticals.

Competing interests MT serves as a consultant and speaker for Eyetech Inc. TRF has also spoken on behalf of Eyetech, Inc.

\section{Patient consent Obtained.}

Ethics approval The study protocol was reviewed and approved by an institutional review board at each study site in accordance with the guidelines for the conduct of clinical research in the 1964 Declaration of Helsinki. The study is listed on www. clinicaltrials.gov (NCT00354445).

Provenance and peer review Not commissioned; externally peer reviewed.

\section{REFERENCES}

1. Gragoudas ES, Adamis AP, Cunningham ET Jr, et al. Pegaptanib for neovascular age-related macular degeneration. N Engl J Med 2004;351:2805-16.

2. Brown DM, Kaiser PK, Michels M, et al. Ranibizumab versus verteporfin for neovascular age-related macular degeneration. N Engl J Med 2006;355:1432-44.

3. Rosenfeld PJ, Brown DM, Heier JS, et al. Ranibizumab for neovascular age-related macular degeneration. N Engl J Med 2006;355:1419-31.

4. Lynch SS, Cheng CM. Bevacizumab for neovascular ocular diseases. Ann Pharmacother 2007;41:614-25.

5. Ferrara N, Damico L, Shams N, et al. Development of ranibizumab, an anti-vascular endothelial growth factor antigen binding fragment, as therapy for neovascular agerelated macular degeneration. Retina 2006;26:859-70.

6. Gordon MS, Cunningham D. Managing patients treated with bevacizumab combination therapy. Oncology 2005;69(Suppl 3):25-33.

7. Tuñón J, Ruiz-Moreno JM, Martin-Ventura JL, et al. Cardiovascular risk and antiangiogenic therapy for age-related macular degeneration. Surv Ophthalmol 2009;54:339-48.

8. Singerman LJ, Masonson H, Patel M, et al. Pegaptanib sodium for neovascular age-related macular degeneration: third-year safety results of the VEGF Inhibition Study in Ocular Neovascularisation (VISION) trial. $\mathrm{Br} \mathrm{J}$ Ophthalmol 2008;92:1606-11.

9. Marcus DM. VEGF Inhibition Study in Ocular Neovascularization Clinical Trial Group. Four-year safety of pegaptanib sodium in neovascular age-related macular degeneration (AMD): results of the V.I.S.I.O.N. trial. Invest Ophthalmol Vis Sci 2008;49:E-abstract 5069.

10. Apte RS, Modi M, Masonson H, et al. Pegaptanib 1-year systemic safety results from a safety-pharmacokinetic trial in patients with neovascular age-related macular degeneration. Ophthalmology 2007;114:1702-12.

11. Hughes MS, Sang DN. Safety and efficacy of intravitreal bevacizumab followed by pegaptanib maintenance as a treatment regimen for age-related macular degeneration. Ophthalmic Surg Lasers Imaging 2006;37:446-54.

12. Farah SE. Treatment of neovascular age-related macular degeneration with pegaptanib and boosting with bevacizumab or ranibizumab as needed. Ophthalmic Surg Lasers Imaging 2008;39:294-8.

13. Nishijima K, Ng YS, Zhong L, et al. Vascular endothelial growth factor-A is a survival factor for retinal neurons and a critical neuroprotectant during the adaptive response to ischemic injury. Am J Pathol 2007;171:53-67.

14. McLeod DS, Grebe R, Bhutto I, et al. Relationship between RPE and choriocapillaris in age-related macular degeneration. Invest Ophthalmol Vis Sci 2009;50:4982-91.

15. Peters S, Heiduschka $P$, Julien $S$, et al. Ultrastructural findings in the primate eye after intravitreal injection of bevacizumab. Am J Ophthalmol 2007;143:995-1002.

16. Papadopoulou DN, Mendrinos E, Mangioris G, et al. Intravitreal ranibizumab may induce retinal arteriolar vasoconstriction in patients with neovascular age-related macular degeneration. Ophthalmology 2009;116:1755-61.

17. UhImann S, Friedrichs U, Eichler W, et al. Direct measurement of VEGF-induced nitric oxide production by choroidal endothelial cells. Microvasc Res 2001;62:179-89.

18. Moncada S, Higgs EA. The discovery of nitric oxide and its role in vascular biology. Br J Pharmacol 2006;147(Suppl 1):S193-201.

19. Verheul HM, Pinedo HM. Possible molecular mechanisms involved in the toxicity of angiogenesis inhibition. Nat Rev Cancer 2007;7:475-85.

20. Bombeli T, Karsan A, Tait JF, et al. Apoptotic vascular endothelial cells become procoagulant. Blood 1997;89:2429-42.
21. Russell DA, Abbott CR, Gough MJ. Vascular endothelial growth factor is associated with histological instability of carotid plaques. Br J Surg 2008;95:576-81.

22. Gillies MC, Wong TY. Ranibizumab for neovascular age-related macular degeneration. N Engl J Med 2007;356:748-9; author reply 9-50.

23. Ueta T, Yanagi $Y$, Tamaki $Y$, et al. Cerebrovascular accidents in ranibizumab. Ophthalmology 2009;116:362.

24. Boyer DS, Heier JS, Brown DM, et al. A phase Illb study to evaluate the safety of ranibizumab in subjects with neovascular age-related macular degeneration. Ophthalmology 2009;116:1731-9.

25. Lalwani GA, Rosenfeld PJ, Fung AE, et al. A variable-dosing regimen with intravitreal ranibizumab for neovascular age-related macular degeneration: year 2 of the PrONTO Study. Am J Ophthalmol 2009;148:43-58.e1.

\section{APPENDIX}

Writing Committee Thomas R. Friberg, University of Pittsburgh (chair and corresponding author); Michael Tolentino, Center for Retina and Macular Disease; Pamela Weber, SUNY at Stony Brook University Medical Center; Sunil Patel, Retina Research Institute of Texas, LLC; Scott Campbell, Eyetech, Inc; Mauro Goldbaum, Eyetech, Inc.

\section{Members of the LEVEL Study Group}

Principal investigators: Prema Abraham, Black Hills Regional Eye Institute, Rapid City, South Dakota; Virgil Alfaro, Retina Consultants of Charleston, Charleston, South Carolina; Andrew Antoszyk, Charlotte Eye Ear Nose and Throat Associates, PA, Charlotte, North Carolina; Laurence Arend, Ochner Health System, New Orleans, Louisiana; Mark Balles, Retina Center of Maine, South Portland, Maine; Charles Barnes, Wolfe Eye Clinic, Cedar Rapids, lowa; C Joseph Beck, Grene Vision Group, Wichita, Kansas; Michael Bennett, Retina Institute of Hawaii, Honolulu, Hawaii; Adam Berger, Center for Retina and Macular Disease, Winter Haven, Florida; Brian Berger, Retina Research Center, Austin,Texas; Todd Berger, Orlick, Berger and Kasper, MD's, PA, Saint Petersburg, Florida; George Bertolucci, Eye Medical Center, Fresno, California; Paul Blacharski, Inland Valley Retina, Corona, California; Steven Bloom, Eye Centers of Louisville, Louisville, Kentucky; Thomas Bochow, Texas Eye Care Associates, Tyler, Texas; Esther Bowie, Storm Eye Institute, Charleston, South Carolina; Richard Breazeale, Southeastern Retina, Chattanooga, Tennessee; Miguel Busquets, Associates in Ophthalmology, West Mifflin, Pennsylvania; John Butler, Retina Consultants of Delmarva, PA, Salisbury, Maryland; Ken Carnevale, Ophthalmic Consultants of LI, Stony Brook, New York; Rangu Chandran, Sylvan Eye Associates, Modesto, California; Nauman Chaudhry, New England Retina Associates, New London, Connecticut; Tom Ciulla, Midwest Eye Institute, Indianapolis, Indiana; Timothy Cleland, Retina Associates of S Texas, San Antonio, Texas; Tom Connor, The Eye Institute/Medical College of Wisconsin, Milwaukee, Wisconsin; Joel Corwin, Miramar Eye Specialists Medical Group, Ventura, California; Scott Cousins, Duke University Eye Center, Durham, North Carolina; Richard Culbert, Premier Retina Specialists, Midland, Texas; David Dodwell, Illinois Retina Center, Springfield, Illinois; Alan Downie, Associated Eye Care, Stillwater, Minnesota; David Dyer, Retina Associates, PA, Shawnee Mission, Kansas; Alexander Eaton, Retina Health Center, Fort Myers, Florida; Charles Eifrig, Retina Associates, Raleigh, North Carolina; John Elfervig, Vitreo Retinal Foundation, Memphis, Tennessee; Michael Elman, Elman Retina Group, Baltimore, Maryland; Robert Equi, Retina Consultants of Sacramento, Sacramento, California; David Faber, Rocky Mtn Retina Consultants, Salt Lake City, Utah; Philip Falcone, Connecticut Retinal Consultants, New Haven, Connecticut; Robert Feldman, Florida Eye Clinic, Altamonte Springs, Florida; Thomas Friberg, UPMC Eye Center, Pittsburgh, Pennsylvania; Martin Friedlander, Scripps Clinic, La Jolla, California; Hua Gao, Indiana University, Dept of Ophthalmology, Indianapolis, Indiana; Enrique Garcia-Valenzuela Midwest Retina Consultants, SC, Arlington Heights, Illinois; David Glaser, Retina Associates of St Louis, Florissant, Missouri; Christine Gonzales, UCLA-Jules Stein Eye Institute, Los Angeles, California; Victor Gonzalez, Valley Retina Institute, PA, McAllen, Texas; Carmelina Gordon, TLC EyeCare and Laser Centers, Jackson, Michigan; Craig Greven, Wake Forest University Eye Center, Winston-Salem, North Carolina; Nicole Gross, Retina Associates of NY, New York, New York; Sunil Gupta, Retinal Specialists, Pensacola, Florida; Larry Halperin, Retina Group of Florida, Fort Lauderdale, Florida; Allen Ho, Associated Retinal Surgeons, PA, Cherry Hill, New Jersey; Henry Hudson, Retina Centers, PC, Tucson, Arizona; Mark Hughes, Ophthalmic Consultants of Boston Stoneham, Massachusetts; Michael Johnson, Eastern Maine Retina Associates, Bangor, Maine; Steven Johnson, Central Coast Eye Associates, San Luis Obispo, California; Brian Joondeph, Colorado Retina Associates, Denver, Colorado; Eric Kanter, Retina Vitreous Consultants, Livingston, New Jersey; Randy Katz, Florida Eye Microsurgical Institute, Inc, Boynton Beach, Florida; John Khadem, Retina Specialist, PC, New York, New York; Faruk Koreishi, Retina Consultants of Western NY, Williamsville, New York; Magdalena Krzystolik, Southern New England Retina Associates, Providence, Rhode Island; Christine Ku, Inland Valley Retina, Corona, California; Derek Kuhl, The Retina Center, Bryan, Texas; Rohit Lakhanpal, Eye Center at Union Memorial Hospital, Baltimore, Maryland; Howard Lazarus, American Eye Institute, New Albany, Indiana; Darma le, Delaware Valley Retina, Lawrenceville, New Jersey; John Lehr, Macgruder Eye Institute, Orlando, Florida; Nicholas Leondary, Retina Vitreous Associates, Toledo, Ohio; Alan Letson, OSU Eye Physicians and Surgeons, LLC, Dublin, Ohio; Mimi Liu, Porter Adventist Hospital, Denver, Colorado; Charles Lyon, Vitreo-Retinal Associates, Shreveport, Louisiana; Dennis Marcus, Southeast Retina 
Center, Augusta, Georgia; Jose Martinez, Austin Retina Associates, Austin, Texas; William Mieler, University of Chicago, Chicago, Illinois; Daniel Montzka, Gulf Coast Retina, Clearwater, Florida; Andrew Moshfeghi, U of Miami/Bascom Palmer, Palm Beach Gardens, Florida; Roger Novack, Retina-Vitreous Assoc Med Group, Beverly Hills, California; Mike Ober, Henry Ford Medical Center, West Bloomfield, Michigan; Dennis O'Connell, Insight Retinal Consultants, Englewood, Colorado; Sunil Patel, Retina Research Institute of Texas, LLC, Abilene, Texas; Scott Pendergast, Retina Associates of Cleveland, Youngstown, Ohio; William Phillips, The Retina Group of Washington, Greenbelt, Maryland; James Powers, Healthy Vision, Dunedin, Florida; Jay Prensky, Pennsylvania Retina, Camp Hill, Pennsylvania; Paul Raskauskas, Retina Consultants Southwest Florida, Fort Myers, Florida; Ramakrishna Ratnakaram, University of Florida, Gainesville, Florida; Diana Reeves, Omni Eye Specialists, Denver, Colorado; William Rodden, Retina and Vitreous Center S0, PC, Ashland, Oregon; Daniel Rosberger, MaculaCare, New York, New York; Richard Rosen, NY Eye and Ear Infirmary, New York, New York; Robin Ross, Retina Vitreous Center, Grand Blanc, Michigan; Nasi Samiy, Retina Institute of the Carolinas, Gastonia, North Carolina; Joseph Schwartz,
Atlantic Retina Center, Salisbury, Maryland; Clive Sell, Associated Retina Consultants, Phoenix, Arizona; Brian Sippy, Rocky Mountain Eye Center, PC, Missoula, Montana; Raymond Sjaarda, Retina Specialists, Baltimore, Maryland; Craig Sklar, The Eye Care Group, PC, Waterbury, Connecticut; Lory Snady-McCoy, Rhode Island Eye Institute, Providence, Rhode Island; Jeffrey Stern, Northeast Retina Associates, Albany, New York; Michael Stewart, Mayo Clinic of Jacksonville, Jacksonville, Florida; Eric Suan The Retina Care Center, Baltimore, Maryland; Jeffrey Taylor, Taylor Vitreoretinal Center, Raleigh, North Carolina; Dave Telander, UC Davis Medical Center, Sacramento, California; David Tom, New England Retina Assoc, Hamden, Connecticut; Robert Torti, Retina Specialists, DeSoto, Texas; Michael Varenhorst, Vitreo-Retinal Consultants and Surgeons, PA, Wichita, Kansas; Pamela Weber, Island Retina, Shirley, New York; John Wells, Palmetto Retina Center, Columbia, South Carolina; Joseph Wilhelm, Michigan Eye Care Specialists, Lansing, Michigan; Carl Wilson, Kaiser Permanente Med Center, Denver, Colorado; Tien Wong, Vitreo Retinal Consultants, Houston, Texas; John Wroblewski, Cumberland Valley Retina Consultants, PC, Hagerstown, Maryland; Timothy You, Orange County Retina Medical Center, Santa Ana, California. 